(

8

9

\title{
Advancing characterization of social diversity in the Wildland-Urban Interface: An indicator approach for wildfire management
}

(1)

Travis B. Paveglio ${ }^{a}$, Max Nielsen-Pincus ${ }^{b}$, Jesse Abrams ${ }^{c}$, and Cassandra Moseley ${ }^{c}$

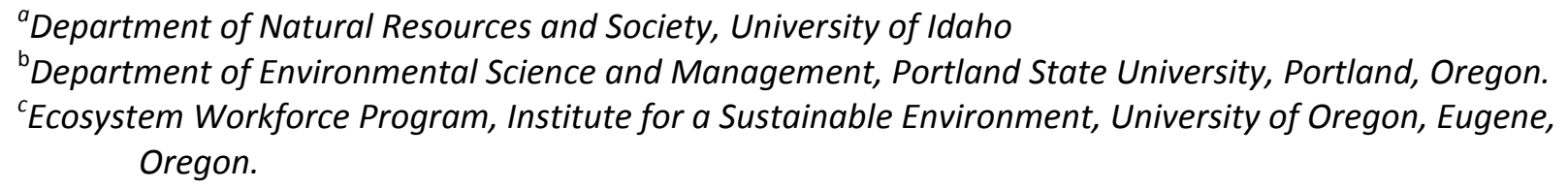

Corresponding Author contact: tpaveglio@uidaho.edu; 509-595-1607; 875 Perimeter Drive, Moscow, ID, USA 83844

Co-author emails: maxnp@pdx.edu; jabrams@uoregon.edu; cmoseley@uoregon.edu

Abstract: A growing body of research indicates that communities at risk from wildfire differ in terms of

the local social context that influences adaptive planning, mitigations or collective actions. Less work has

attempted to document critical differences in that local social context across large samples. The

research presented here explores a quantitative operationalization of an established framework for

characterizing the social diversity of communities at risk from wildfire. We conducted structured

interviews with key informants across nine U.S. states. Factor analysis, regression and hierarchical

26 cluster analysis were used to characterize social context across communities and relate it to key

27 informant evaluations of progress toward fire adaptation. Our results advance methods to

systematically document how social context influences local wildfire adaptation by: (1) examining a

(C) 2016. This manuscript version is made available under the Elsevier user license

http://www.elsevier.com/open-access/userlicense/1.0/ 
preliminary set of quantitative key-informant measures for gauging social context across a range of WUI

communities; (2) identifying related elements of social context that may collectively influence wildfire adaptations; (3) providing preliminary statistical evidence that highly related elements of local social context are correlated with expert assessment of local populations' adaptations to wildfire; and (4) identifyingdifferences in social context characteristics across a sample of western USA WUI communities. However, it also is important to recognize that the measures tested here serve as indicators of deeper conceptualunderstandings informed by in-depth case studies. Efforts to use these measures should be augmented with additional qualitative work and build from those deeper understandings by considering the complexity of local dynamics surrounding wildfire management.

Keywords:Fire adapted community; wildfire; hazard planning; social context

\section{Introduction}

The need for human adaptation in the face ofgrowing risks associated with wildfireis a welldocumentedimperative (Pyne 2015, Toman et al. 2013). Current approaches to wildfire adaptation in the United States and elsewhere are aimed largely at fostering or advancing human populations that can effectively prepare for, respond to, and recover from the disturbance caused by wildfire while reintegrating it as a component of healthy ecosystems (FACC 2015).In the United States, this goal has recently been subsumed under the broad heading of creating "fire-adapted communities" (FACs). FACs are encouraged to adopt, enact, and modify a variety of previously developed programsand planning strategies that focus on actions individual homeowners, communities, and fire professionals can take to 
52 make human populations and infrastructure more capable of "living with fire" (WFEC 2014; Paveglio et 53 al. 2016)

FACs are the latest development in a long-term focus on fostering private individual and community responsibility for wildfire management while integrating wildfire planning and response obligations across jurisdictions (e.g., city, county, state, federal agencies) (McCaffrey and Olsen 2012; Jakes and Sturtevant 2013;Abrams et al. 2015).The FAC concept acknowledges that many mitigation actions are most effective when they are performed consistently across populations thatshare risk from wildfire, and that collective planning among such populations can leverageresources to better help individuals addresswildfire fire impacts (Kyle et al. 2010; Brenkert-Smith 2010).The focus on collective or consistent mitigations among private citizens is a response toincreased damage and impacts from wildfire to human populations and the rising governmental costsassociated with suppressing wildfire to protect private property or other human values such as ecosystem services (e.g., watersheds, timber, and recreational opportunities) (Nielsen-Pincus et al. 2015; Paveglio et al. 2015a). proven to be a significant challenge.Comparisons of social science efforts have foundthat levels of support forand perpetuation of adaptive actions designed to address wildfire risk are inconsistentacross study populations and time (Paveglio et al. 2015b; Stidham et al. 2014; Meldrum et al. 2014).Researchers have not identified a consistent set of correlates to consistently predict or promote adoption of private actions that reduce wildfire risk (Cohn et al. 2008; Jakes and Langer 2012; Kulig et al. 71 2013).

A number of scholars and policymakers have recognized the need for a diverse range ofwildfire 73 adaptation strategies that can be applied selectively to socially distinctpopulations (Fischer et al.

74 2012;Steelman and McCaffrey 2013). A first step in tailoring wildfire adaption strategies is the 75 development and exploration of existing frameworks for documenting critical differences in social 
context that vary across populations. Existing frameworks are built from in-depth data that recognizes

77 how area-specific social context is likely to influence the variable wildfire adaptations most likely to be effective in promoting personal or collective responsibility among those populations (Paveglio et al.

2015b; Stidham et al. 2014; Brenkert-Smith 2011). However, there has not been much effort to operationalize or quantitatively explore the conceptual relationships of those frameworks across larger samples of human populations to better make sense of diversepopulations across regions (Spies et al. 2014; Ager et al. 2015).

The research presented in this paper advances conceptual and methodological efforts to understand how best to foster FACsbyexploring aquantitative operationalization of an established framework for documenting the social diversity of communities at risk from wildfire (Paveglio et al. 2009; 2012; 2015b).Our intent is to make progress toward a quantitative approach that investigates howunique elements of social context are likely to differentially influence local approaches to and support for collective organizing to address wildfire risks and wildfire mitigation strategies across landscapes.That more rapid quantitative approach could be expanded and paired with existingconceptual, in-depth efforts for a more comprehensive picture of how local social context influences efforts to foster locally tailored planning, programs and actions that diverse populations can use to adapt to wildfire in ways that match their unique socio-ecological conditions.

\subsection{Adapting to wildfire}

Creating fire-adapted communities is one of three broad goals outlined in the National Cohesive Wildland Fire Management Strategy, which presents a national vision to guide present and future wildfire management activities and policy in the United States (WFEC 2014). Yet understandings of what makes communities "fire adapted" are still emerging. In many ways, the concept of "fire-adapted communities" has helped organize a variety of existing programmatic and policy approaches that 
attempt to foster private citizen, community, and governmentparticipation in local wildfire adaptation (e.g., wildfire management, planning, mitigation) at a variety of spatial and temporal scales.For instance, the Fire Adapted Communities (FAC) Program advocates Firewise mitigation principles that homeowners can conduct around their homes (e.g., clearing of trees and brush near the home, using fire resistant building materials) in order to reduce structure flammability and the need for professional suppression efforts (FAC 2015; NFPA 2015). Likewise, the FAC program highlights the importance of Community Wildfire Protection Plans (CWPPs)that promote collective, long-term planning for wildfire risk, prioritization of large-scale fuel reduction projects, and coordinated emergency management during wildfires (Williams et al. 2012).

Existing research has identified a number of variables as influential in the promotion of adaptive wildfire actions among at risk populations, including those outlined in the Firewise program: (1) primary or secondary homeownership; (2) awareness of and perception concerning wildfire risk in the locality; (3) available income; (4) perceived efficacy of actions to reduce local fire risk; (5) voluntary or mandated actions; (6) community attachment; (7) trust in land and wildfire managers; and (8) perceived impact on aesthetic or other values (Busby et al. 2013; Absher and Vaske 2011; Meldrum et al. 2014; McCaffrey et al. 2011; McCaffrey 2015). Other wildfire research also suggests that the flexibility of planning, decisions about the scale of planning initiatives, involvement of consultants, collaborative goal setting for fuels reduction, and community participation influence the enactment and perpetuation of CWPPs (Williams et al. 2012; Abrams et al. 2015). While each of these aspects may be important, none of them have been shown to consistently drive the enactment of adaptive actions across all or even the majority of populations at risk from wildfire.

One theme that continues to emerge across wildfire and broader hazardresearchis the high degree ofvariability in social and ecological conditions of populations at risk from disturbance events (Paveglio et al. 2009; Gordon et al. 2012; Collins et al. 2012; Sword-Daniels et al. 2016). This variability 
124 can have asignificant influence on the strategies enacted or supported by local people in their

125 adaptation to changing wildfire risks or other hazards (Brenkert-Smith 2011; Nielsen-Pincus et al.

126 2015;Petty et al. 2015; Paton et al. 2015).Ongoing research in the WUI hasdemonstrated that human

127 populations living there can have vastly different histories, perspectives, local cultures and means of

128 organizing (McCaffrey et al. 2011; Paveglio et al. 2015b).

The social diversity of WUI communities at risk from wildfirehelps explainwhy common factors

130 influencing individual or community adaptation may be more relevant or salient in some places than

131 others.For instance, commonly mentioned correlates for performance of risk reduction actions vary

132 across distinct populations, including: (1) risk perception; (2) available income; (3) scale of planning; (4)

133 trust in land management agencies; and (5) concern about landscape health(Sharp et al. 2013; Dickinson

134 et al. 2015; Champ et al. 2015; McCaffrey et al. 2015).Likewise, research also indicates that diverse

135 communities at risk from wildfire will collectively support, perpetuate or benefit from different

136 community-scale strategies (e.g., collective fuel breaks, strengthening rural fire protection districts) for

137 wildfire planning and protection (Dunlop et al. 2014; Paveglio et al. 2012; Paveglio2016). The growing

138 body of such findings suggests that while common influences on mitigation actions are important, it also

139 is critical to consider how both individual-level and broader considerations (e.g., local community) bear

140 on adaptive actions. Likewise, it may be more useful to examine the circumstances that will cause

141 common correlates to vary in their influence on adaptation across populations (Brenkert-Smith 2010;

142 Gordon et al. 2012). Individual-level actions such as household fuels reduction or evacuation planning

143 may be influenced by community norms, values or proliferation of information through local social

144 networks, while collective actions may hinge on whether individuals choose to identify with or

145 contribute to "communities" of individuals performing similar actions (e.g., collective prioritization of

146 fuels reduction, volunteering for rural fire districts) (Martin et al. 2009; Brenkert-Smith 2010; Kyle et al.

1472010 ). That is, characteristics of local context interact. Existing studies often operationalize each 
148 influence (i.e., variable) as a separate correlate or predictor of some adaptive action. Limited effort has

149 been made to explore interactions or relationships between characteristics to provide a more holistic 150 view of the local context that drives adaptation (Paveglio et al. 2016).

In sum, existing wildfire science and risk research stress that it is important to first understand

152 the people and area culture when determining what motivates or influences the ways they respond to 153 risk (Short 1984; Flint and Luloff 2005; Steelman and McCaffrey 2013).Communicating new strategies for

154 adapting to wildfire (or any risk), and collaborativelydeveloping those strategies with local

155 populationsrequires tailored approaches that recognize differences in the local social context that

156 influences those people (Paveglioet al. 2012; Fischer et al. 2012; Collins 2012). The outcome of risk

157 management strategies better tailored to local context is likely to be divergent combinations of adaptive

158 actions and increased levels of support, adoption, or perpetuation of risk reduction activities because

159 the actions are more likely to become part of local culture (Jakes et al. 2011; Monroe et al. 2015). We discuss existing efforts to understand local social context relative to wildfire risk in the next section.

1.2 Community characterization and wildfire adaptation

164 Researchers and policymakers have long called for better characterization of social diversity in

165 communities at risk from hazards, and specifically for WUI communities (Lee 1991; Jakes et al. 2007).

166 Some wildfire researchers recently adopted methodologies and frameworks from hazard and climate

167 changeliteraturesto characterize communities'differential "social vulnerability" to wildfire(see Poyudal

168 et al. 2012;Solangaarachchi et al. 2012;WFEC 2014 for recent examples). These studies pair

169 selectdemographic data and measures of exposure to wildfire risk (often probabilistic simulations of fire 170 occurrence or spread) to determine the potential for wildfire (i.e., exposure) and the magnitude of

171 potential fire impacts (i.e., sensitivity) to those populations. Demographic characteristics such as age, 
172 income, education and race continue to be used as proxies for levels of potential exposure or sensitivity

173 (Ojerio et al. 2011; WFEC 2014;Johnson Gaither et al. 2015). While these efforts are insightful, they may 174 not paint a full picture of the influences on variable vulnerability and adaptive response to wildfire. For 175 instance, they have been criticized for:(1) failing to operate at the geographical scales that best 176 characterize socially diverse human communities; (2)employingsecondary data or indicatorsthat have 177 not been tested for wildfire; and (3) inconsistent relationships of correlates with vulnerability across 178 populations(Paveglio et al. 2015b; Paveglio et al. 2016). A separate tradition of research surrounding wildfire planning and mitigation uses primary data 180 at a variety of scales (e.g., individual perceptions, performance of planning, county or state policies) to document the variability of social influences on adaptation across cases (Jakes et al. 2007; Brenkert-

182 Smith 2011; Toman et al. 2013). Paveglio et al. (2009; 2012) adapted case study data and lessons from 183 existing research on wildfire, climate change, and rural/natural resource sociology to provide a 184 framework for documenting social diversity in the WUI. The four broad conceptual categories of that 185 framework provide a means to organize characteristics that mayinteract to differentially influence 186 response to wildfire risk. Those broad conceptual categories are: (1) residents' knowledge of the local 187 ecosystem and experience with wildfire (place-based knowledge); (2) access and ability to adapt scientific/technical information to a local context; (3) demographic (e.g., median income, age and 189 ethnicity) and structural (e.g., road infrastructure, building materials and access to 190 resources)characteristics of local populations; and (4) interactions and relationships within the 191 community that support (or fail to support) collective action. Paveglio et al. (2010; 2012; 2016) have since used existing and ongoing wildfire research across 193 the U.S. West to document 22 specific local characteristics, organized within four conceptual 194 categories, that are likely to influence local community support for, establishment, and ongoing 195 performance of wildfire adaptations across populations. These characteristics represent lessons from 
both the "social psychological" tradition of wildfire social science that focuses on individual-level or

197 perceptual factors that influence adoption of wildfire mitigations and the "process" tradition that

198 focuses on the place-based interactions among people, including collaborative planning or organizing

199 that foster collective wildfire adaptation actions (Paveglio et al. 2015). Many of these characteristics are

200 difficult to ascertain using existing secondary data. Paveglio et al. (2015)compared 18 case studies of

201 community response to fire risk using the22 local characteristics to outline a typology of four different

202 "archetype" WUI communities that likely will respond to risk and efforts to reduce it in characteristic

203 ways. The fourarchetypes include:(1)formalized suburban WUI; (2) high amenity, high resource WUI; (3)

204

205

206

207

208

209

210

211

212

213

214

215

216

217

218

rural lifestyle WUI; and (4) working landscape/resource dependent WUI.Identification of archetype communities are intended to promote FACs by providing diverse stakeholders with a means to more quickly identify the unique conditions operating in a given community and develop tailored approaches to wildfire adaptation that they can share among communities with similar context.

Paveglio et al.'s (2009; 2012; 2015) approach focuses on the unique combinations of local social characteristics and external forces (including ecological processes or larger social forces) that influence whether and how human communities take action to reduce their exposure or modify their severity of disturbance events (Norris et al. 2008; Ford et al. 2010). Central to those processes are the effect that local culture has on fostering or reinforcing individual action, the historic and ongoing influence locals' relationship with the landscape (e.g., resource extraction, amenity migration) has on their perspectives, and the ways that residents create risk through their common values or attitudes (Flint and Luloff 2005; Flint et al. 2010).Thus, the approach acknowledges the multi-level structure of influences on wildfire adaptation, but focuses at the scale of shared commitment or performance of collective action. The key to Paveglio et al.'s approach $(2009 ; 2012 ; 2015)$ centers on the way that adaptationemerges from the interaction of select characteristics operating in each locality studied and the variability of potential 
adaptation(both in support for actions and the type of actions populations are likely to enact) across cases based on community-specific context.

222 Collins 2012; Gordon et al. 2012)have largely been qualitative in nature.Thus, they are restricted to a

223 relatively small number of comparisons across populations as required by the intensity of an approach

224 that seeksan in-depth conceptual understanding as its primary goal. Those same efforts also can be

225 time-consuming and lacking generalizability because they are site-specific. In response, there have been

226 calls to apply a uniform methodology to quantify whether orhow elements of social context might

227 combine to take unique forms across diverse WUI communities and how those combinations influence

228 different adaptive wildfire actions. This includes common measures that can help understand the

229 magnitude of characteristics and their influences on adaptation across populations (Ojerio et al. 2011;

230 Adger et al. 2015). Development of such an approach provides a different means to examine the conceptual relationships or frameworks that call for differential strategies for wildfire adaptation.

Although social context may vary from one community to another, we hypothesize that common profiles of local social context exist that can be used to inform efforts to develop FACs. To this end, our objectivesrespond to emerging research and policy needs by exploringPaveglio et al.'s approach (2015b) using quantitative key informant ratings of social context in a broader sample of western U.S. communities than has been conducted previously. We use this assessment to: (1)

237 quantitatively explore the relationships between elements of local social context in WUI communities,

238 (2) identify the influence that elements of local social context, and their interactions, have on local 239 adaptations to wildfire, and (3) to better understand whether we can use quantitative, key informant 240 assessments to document differences in social context and their relation to wildfire adaptation across 241 populations. 


\section{Methods}

$244 \quad 2.1$ Key informant sampling and structured interviews

We used structured interviews with key informants to assess the local social context likely to

246 influence wildfire risk adaptationoperating in their communities. Structured interviews are a common

247 method of administering a survey instrument to multiple individuals in geographical diverse localities

248 (Bryman 2012). Key informants are those individuals who have specialized knowledge of or experience

249 with the subject of interest for a given context, in our case a comprehensive understanding of local

250 context influencing local wildfire adaptation. Key informant approaches have long been used in

251 community research and research on preparation for hazards or risk (see for instance Krannich and

252 Humphrey 1986; Eyler et al. 1999; Sherrieb et al. 2012).

Developing a sample frame of individuals for the structured interviews first necessitated

254 selection of diverse geographic locations with existing and future wildfire risk.It was part of a larger

255 effort to identify diverse study populations who had engaged in processes of adaptation for wildfire. We

256 began site selection by identifying countiesthat had recently experienced wildfires using a database of

257514 wildfires that: (1) occurred between 2004 and 2012 in ten western United States; (2) for which the

258 US Forest Service was the lead suppression agency; (3) resulted in more than \$1 million in federal

259 suppression expenses; and (4) that had completed a CWPP that could be found online. We targeted

260 counties withpopulations of less than 250,000 to focus on non-metropolitan counties. Additional sample

261 refinement included selecting only those countiesin which ICS209 daily situation reports (USDA Forest

262 Service 2015) indicated that wildfires threatened or damaged structures or where wildfires forced

263 evacuations. These criteria excluded remote areas with little human habitation and ensured adequate

264 social context for researchers to characterize. The selection criteria above resulted in the identification

265 of 68 counties in nine western U.S. states (Arizona, California, Colorado, Idaho, Montana, New Mexico,

266 Oregon, Utah, Washington) that experienced 144 wildfires. 
We used a combination of theoretical and snowball sampling in the selection of participants for

268 structured interviews. Theoretical sampling concerns selection of individuals who have specialized

269 knowledge about a topic or area of inquiry (Charmaz 2000; Bryman 2012). Snowball sampling is a

270 complimentary strategy that uses chain referral for the identification of additional informants from an

271 initial sample (Linlof and Taylor 2010). Our selection of participants occurred in two stages, namely: (1)

272 contacting leaders and professionals across the 68 countiesto suggest community level contacts; and (2)

273 vetting of additional suggestions of community level contacts who could serve as key informants. We

274 explain details of this process below.

275

At the county scale, we sought professionals involved in CWPP development or implementation

276 (e.g., fire chiefs or fire marshals, emergency operations managers and county planners). We successfully

277 contacted CWPP planners and managersin52 counties, who were then asked to recommend two to

278 three community-level professionals or key informants with direct experience and knowledge about

279 communitiesmost affected by past local wildfires.County-level contacts identified 137 community-level

280 individuals who ranged widely in terms of their affiliation, including firefighting professionals, city

281 government officials, land management agency representatives, emergency managers, and leaders of

282 local civic groups.

Community-level respondents were contacted via telephone for aninterview comprised of both

284 structured (closed-ended) and semi-structured (open-ended) questions. Telephone interviewers were

285 provided with a detailed protocolto minimize bias across survey responses. Key informants were

286 contacted up to six times via telephone and email before being removed from the contact list.

287 Respondents who did not self-identify as "knowledgeable" or "very knowledgeable" about wildfire 288 planning, wildfiremitigation and social dynamics in the area studiedwere asked to provide contact

289 information for more knowledgeable participantsfor the community and their responses were

290 discarded. This snowball sampling process occurred until no further respondents could be reached. 
Each interview was treated as an independent sample because each informant was allowed to

292 define their "community" and because the data obtained best represents the key informant's expert

293 perceptions. There is a long tradition of using expert, key informant perspectives to represent

294 organizational or community conditions because few individuals in any given area may have the

295 comprehensive knowledge needed to assess relevant social context (Wagner et al. 2010; Homburg et al.

296 2012). A total of 115 community-level key informants completed the interview protocol for a response

297 rate of $84 \%$. Our selection process (described above) ensured that the sample included representation

298 of expert key informants, including our two-stage sampling process and self-confirmation as

299 knowledgeable respondents. Interviews typically lasted between 30 and 60 minutes. Closed-ended

300 responses were recorded directly into an online database. A breakdown of respondents by state is

301 provided in Table 1.

302

303

2.2. Structured interview survey measures

Elements of social context likely to influence wildfire adaptation were measured in the key

305 informant interviews by asking respondents to rate 41 statements organized under 6 overarching questions prompts. Each statement was evaluated by the respondent using a 5-point Likert-scale that ranged from strongly agree (+2) to strongly disagree (-2), with a mid-point anchor labeled neither agree nor disagree(see Appendix 1).Overarching prompts asked each respondent to evaluate how well each 309 statement reflected their community dynamics, resident sentiments about wildfire, resident preparation 310 for wildfire, risk perceptions, firefighting resources, and the business and civic climate in their 311 community with respect to wildfire. The 41 statements were designed to reflect the 22 social context 312 characteristics described by Paveglio et al. $(2012 ; 2015 b)$ as potentially influencing wildfire adaptation in 313 diverse communities. Multiple statements were often created to fully represent the complex 
314 characteristics identified by those authors (e.g. local peoples' experience with wildfire) and encompass 315 the subthemes they describe.

Additionalstatements (i.e., beyond the 41 outlined above)were designed to elicit respondent

317 assessments regarding the progress the community had made in adapting to wildfire risk. Topics

318 covered included evaluation of community organization surrounding wildfire riskand implementation of

319 publicity campaigns regarding wildfire risk.These statementsalso used the strongly agree to strongly

320 disagree Likert-scale described above and were designed to serve as preliminary indicators regarding

321 how differential elements of social context influence adaptive actions.

\subsection{Data analysis}

All analyses were conducted in the quantitative analysis software SAS 9.3 (SAS Institute, Cary,

$325 \mathrm{NC}$ ).We began withan exploratoryfactor analysis of the social context items. Factor analysis was selected as an initial analysis strategy because it could help us begin to identify the elements of social context

327 that have significant relationshipsbefore applying a smaller number of those variables to some

328 predictive relationship. Likewise, the correlation structure between items that load on a common factor 329 provides some indication that they mayshare a common antecedent in the way described by Paveglio et 330 al. $(2009 ; 2015)$ as qualitative "interactions" among local elements of social context.A version of 331 thefactor analysis was performed using an iterated principle factor method with both an oblique and 332 varimax rotation, keeping all those factors with an initial eigenvalue greater than 1.0 (this was supported 333 by a natural visual break in the scree plot of the eigenvalues) (Stevens 2009). Comparison of the varimax 334 and oblique rotation outputs showed no significant difference in the factor loadings or factors identified, 335 and we proceeded with the varimax rotation. We created an index for each dimension identified by the factor analysis. Items with factor loadings of at least 0.40 were used to create an index for each 
dimension. Indices were createdby averaging responses to the items that comprised each factor.Indices

were assessed for internal validity using a Cronbach's (1951) alpha criterion of at least 0.70.

Another goal of this research was to make progress in determining how different elements of social context might influence select adaptive actions. We employed multivariate regression to explore the influence of respondents' ratings for each social context index on key informant assessments about the relative success ofselect adaptive actions to address wildfire risk in the locality. We first examined the main effects of the social context indices on key informant assessments of local wildfire adaptation, and then included first-order interactions between indices of social context to explore whether interaction between indices of social context further explains relationships to perceived performance oflocal wildfire adaptation.

Our final research goal was to explore whether key informants could be grouped into common expressions of social diversity influencing adaptive actions in the WUI. We used a hierarchical cluster analysis using the Ward's minimum-variance method (Ward 1963). Hierarchical cluster analysis is a wellknown method for reducing anumber of disparate observations into a smaller set of categories using multivariate criteria (Stevens 2009). In this case, the results of the factor analysis and resultant indices served as those criteria. We applied a linear transformation of the index values prior to running the cluster analysis using the approximated within-cluster covariance estimation technique (Everitt 1980) to improve cluster classification. Clusters were identified using heuristic guides for the R2, Pseudo F, and Pseudo T2 statistics and visual examination of the cluster hierarchy tree (Everitt 1980). We used ANOVA to test for the existence of mean differences in social context indicesamong the cluster groups. We then used the Tukey-Kramer HSD test to estimate and test for differences among each group means (alpha=0.05).

\section{Results}




\subsection{Community social context}

Key informants tended to agree with most of the social context items, and generally provided a positive view of their communities. Only two items had mean values that were significantly less than zero (indicating that respondents, on average, tended to disagree with the item), and nearly threequarters of the items had mean values that were significantly greater than zero (see Appendix 1). Informants tended to disagree that their community had an active local biomass or wood products

367 industry and that the community had sufficient resources to manage wildfire risk. In contrast, 368 informants agreed most strongly that members of theircommunity had family history, knowledge about 369 the local ecology, respected leadership, and people with emergency management and wildland fire 370 experience.

Initial exploratory factor analysis of the social context items revealed four underlying

372 dimensions of the data.This first iteration of our factor analysis resulted in18 items that did not load 373 substantially on any of the four factors. Those items were removed from the analysis and the factor 374 analysis was re-run. The second iteration of the factor analysis resulted in a four-factor solution that 375 explained $56 \%$ of the variance in the data. However, we were interested in retaining only the most 376 strongly correlated characteristics for each factor, and removed an additional 11 characteristics based 377 on natural breaks in the magnitude of factor loadings. The final four-factor solution accounts for $66 \%$ of 378 the variance in the data, provided relatively interpretable factors with no substantial cross-loadings, and 379 best met our goal of identifying the most strongly correlated characteristics of social context (Table 2). For the sake of clarity and simplicity, we labeled the four highly correlated factors of social

381 context characteristics for wildfire as: (1) social cohesion; (2) place-based knowledge; (3) access to 382 scientific and technical knowledge; and (4) shared responsibility and understanding. Cronbach's alpha 383 statisticsfor these factors range from 0.70 to 0.89 , with social cohesion among locals and access to 384 scientific and technical knowledge demonstrating the highest reliability ratings. 
context elements and critical linkages to other realms of community context. For example, actionslocals could take to manage wildfire risk werehighly inversely correlated with the degree to which residents considered wildfire risk to be someone else's problem. Put in another way, key informants responses indicate that local knowledge is associated with more local responsibility for wildfire risk.

Results from main effects regression analysis using the factor indices accounted for a relatively high proportion of variance among key informant ratings for effective community organizing to address 396 wildfire risk $\left(R^{2}=0.45\right)$ and implementation of a publicity campaign about reducing wildfire risk on 397 private property $\left(R^{2}=0.28\right)$. A second set of regression analyses that included interaction effects across 398 factor indices moderately, but significantly $(p<0.001)$, increased the explanatory power of both models $399\left(R^{2}=0.50\right.$ for effective community organizing and $R^{2}=0.36$ for implementation of a publicity campaign). Dependent variable ratings for adaptive actions serve as limited indicators of community adaptation,

401 and influences on these indicators of community adaptation are interpreted through the main and 402 interactive effects among social context indices. 
and access to scientific and technical knowledgesignificantly influence the relationships of both

410 predictors relative to respondent ratings of local organizing effectiveness. More specifically, when there

411 are higher levels of either reportedplace based knowledge or access to scientific and technical

412 knowledge, there is a significant negative relationship between the other predictor andratings of

413 effectiveness for local organizing $(\beta=-0.31 ; p<0.05)$. The standardized effect of reported access to

414 scientific and technical knowledge was $0.34+(-0.31 *$ place-based knowledge and experience), and the

415 standardized effect of reported place-based knowledge and experience was $0.80+(-0.31 *$ access to

416 scientific and technical knowledge). Key informants' ratings of effective community organizing were also

417 significantly influenced by interactions between the social cohesion index and the place-based

418 knowledge and experience index $(\beta=0.50, p<0.05)$. Accordingly, the social cohesion index lost

419 significance as a predictor in the interaction effects model, while access to scientific and technical

420 information explains less variance in the dependent variable $(\beta=0.34, p<0.05)$. The significance of place-

421 based knowledge increases in the interaction effects model, as does the amount of variance explained

422 by that index $(\beta=0.80, p<0.01)$

423 Access to scientific and technical knowledge had a significant positive relationship with key

424 informant ratings concerning the implementation of wildfire publicity campaigns in the main effects

425 model $(\beta=0.52, p<0.001)$ (Table 3). We found a significant interaction effect between the access to

426 scientific and technical knowledge index and the shared responsibility index in the interaction effects

427 model $(\beta=-0.42 ; p<0.05)$, indicating that as either access to scientific knowledge and technical

428 knowledgeorshared responsibility and understanding increase, there is a significant negative

429 relationship between the other predictor and ratings about implementation of publicity

430 campaigns.Accordingly, shared responsibility and understanding became a moderately significant

431 predictor in the interaction effects model $(\beta=0.60 ; p<0.05)$, while the variance explained by access to

432 scientific and technical knowledge increased $(\beta=0.64 ; p<0.001)$. 
Cluster analysis of the social context indices derived from key informant ratings supported four

437 solution was 44\%, with the CCC, Pseudo F, and Pseudo T2 statistics indicating adequate solutions at 438 either 3,4 , or 5 clusters. We selected the 4 cluster solution because further disaggregation of clusters explained relatively little additional variance, and because the four cluster solution provided substantial numbers of observations in each cluster without causing one cluster to dominate. The number of observations in each cluster (Table 4, Panel A) ranged from 21 (18\%, Cluster 4) to 48 (42\%, Cluster 1). ANOVA results revealed significant differences in social context index scores across the clusters

443 (Table4 Panel B). Shared responsibility and understanding varied most widely across the clusters, and 444 had the largest magnitude of difference among clusters followed by both place-based knowledge and access to scientific and technical knowledge. Social cohesion among clusters was the most consistent among clusters. residents. There is less indication that second homeowners are present or willing to address wildfire risk, that people understand the lack of resources for protecting all homes from wildfire, or thatplace-

450 based knowledge is strongly present. Cluster 2 scores relatively highly on all four indices. It has the 451 highest rating of any cluster for place-based knowledge, social cohesion, and shared responsibilities for 452 wildfire among residents. Cluster 3 scores relatively low on all four indices. It is strongest in social 453 cohesion, butscored the lowest on this index among all the clusters. Cluster 4 is moderate in shared 454 responsibility and understanding and social cohesion among residents, but scores the lowest of all clusters on place-based knowledge and access to scientific or technical knowledge. 


\section{Discussion}

The purpose of this research effort was to explorereplicable methods for characterizing the social diversity of WUI communities at risk from wildfire from a large sample of communities. We operationalized a limited application of an existing conceptual framework based on social context characteristics likely to influence wildfire adaptation in order to assess some of its conceptual properties. Our efforts advance methods to systematically document how social contextinfluences progress toward FACs in a number of ways, including: (1) development and explorationof a preliminary set ofkey-informant measures that professionals could use to gaugesocial context across a range of WUI communities; (2) identification of highly related elements of social context that maycollectively influenceselect wildfire adaptations; (3) preliminary statistical evidence that those highly related elements of local social context are correlated with expert assessment of local populations' adaptations to wildfire; and (4) assessment of differences in social context characteristics across a sample of western USA WUI communities.

Factor analysis of key informant ratings suggest that existing conceptual dimensions of social context have continued merit as a means to conceptually organize elements of local dynamics potentially influencing collective wildfire actions (Paveglio et al. 2009; 2012; 2015). Adaptive wildfire actions are not dictated by any one or a select few predictors, they are the collective product of many local context factors that collectively influence how people respond to changing risks such as wildfire (Jakes and Langer 2012; Jakes and Sturtevant 2013; Paveglio et al. 2016). Results of our factor analysis support these ideas, and emphasize that it is important to determine how interrelated aspects of local context combine to influence different actions. The particular name given to those groupings ultimately is less important than the narrative those insights provide. For instance, our regressionsindicate a strong interaction between respondents' ratings of local knowledge about area ecology (what Paveglio et al. 2012 grouped into the conceptual category of place-based knowledge) and other factors that they 
481 implicated as important for adapting or using scientific and technical knowledge (see also Jakes et al.

482 2007; Jakes et al. 2011 and Diaz et al. 2016 for related discussions).

The output of our analysis does not imply that other elements of social context do not

contribute to collective adaptive actions for wildfire, or have an indirect effecton professionals' ability to

485 predict adoption of certain mitigation actions. The importance of local context also is dependent on the

486 adaptive action being assessed, as different elements of local social context may influence specific

487 actions.For those reasons, it would be unwise to assume that characteristics not retained in our factor

488 analysis or implicated in our regressions do not have an influence on wildfire adaptation or people's

489 capacity to deal with wildfire risk. Rather, these characteristics serve as a broad corpus of influences that

490 can help explain differences in fostering FACs across cases. Likewise, it is difficult for us to compare our

491 factor indices against existing conceptual categories (e.g.,Paveglio et al. 2012; 2015) because they were

categorizing potential elements of social context that would combine to influence sets of linked actions

or planning efforts to reduce wildfire risk, while we focused on a few limited predictors.

Our preliminary analysisidentifies what we might call "first-order" interactions of social context

495 that are likely to collectively influence select wildfire adaptations. They can help provide a smaller

496 number of variables that can be used in larger sample studies as initial or limited indicators of diverse

497 social context. The full body of characteristics used here should be replicated across a range of adaptive

498 actions for creating FACs (e.g., CWPP plan components, detailed measures of mitigations performed by

499 residents, incidence or capacity of rural fire districts) to provide a fuller understanding of adaptation

500 based on social context. We now turn to specific findings from our analysis, which provides both

501 conceptual and practical insights on example adaptive actions.

Specific results from our multivariateregression analysis point toward the critical importance of place-based knowledge and access to scientific and technical knowledge in community organizing for

504 wildfire planning. Our factor of access to scientific and technical knowledge reinforces the importance of 
local leaders (or "sparkplugs") who can motivate collective action, a diverse set of existing skills locals

506 can leverage in sustaining their collective efforts, and a linkage to important local understandings of

507 area ecology (Paveglio et al. 2012; Kuliget al. 2013; Toman et al. 2013). Similarly, our factor of place-

508 based knowledge combines a set of ideas that have all been implicated in existing wildfire research as

509 important influences or precursors to the promotion of adaptive action surrounding wildfire risk,

510 including: local understanding of wildfire risk, knowledge of its role as a landscape process, the

511 mitigation actions most likely to reduce wildfire risk, and a personal responsibility for contributing to risk

512 mitigation efforts (Meldrum et al. 2014; Champ et al. 2015; Diaz et al. 2016). While each of these

513 characteristics have been identified as important contributors to adaptive wildfire actions, our findings

514 indicate that they reinforce one-another and provide an increased understanding of how to achieve

515 broader outcomes.

516 We included interactions among social context indices during our regression analyses to explore

517 whether it is important to consider the contingent influence of local social characteristics on each other

518 in promoting collective wildfire adaptations. We found that some of these interactions were significant

519 correlates with respondents' ratings of select adaptation actions. We also found that the interaction

520 between ratings of factors modified their importance as influences on limited adaptive actions. Perhaps

521 more interestingly, we found thatinteractions among characteristics of local context might not positively

522 influence all wildfire adaptions. Publicity campaigns were more likely where access to scientific and

523 technical knowledge was rated highly but where low ratings of shared responsibility for wildfire risk and

524 limited understanding of fire suppression existed, and vice versa. We also found that higher ratings of

525 both place-based knowledge and access to scientific and technical knowledge among a given

526 populationwere correlated with lower evaluations of local effectiveness in organizing for wildfire.

527 Evaluations of local effectiveness in organizing for wildfire increased when place-based knowledge was

528 high and access to scientific and technical knowledge decreased, or when access to scientific and 
technical knowledge was high and place-based knowledge decreased. Existing wildfire and other hazard

530 research helps explain possible interpretations of thislatter result, namely that people with local experience may have less need to seek out or have less trust inoutside knowledge, instead relying on

532 their existing experience. A community that actively engages with and uses scientific or technical

533 expertise may be doing so because they currently lack the place-based knowledge needed to reduce

534 wildfire risk (Cohn et al. 2008; Sharp et al. 2013; McCaffrey 2015). On the other hand, the interaction

535 between social cohesion and access to scientific and technical knowledge was positive, indicating that

536 community organizing effectiveness rises when people share a sense of community and are able to

537 engage scientific and technical expertise and adapt it to their local context.

approachesfor organizing WUI communities using similar "groupings" of local social context (what

540 Paveglio et al. 2015identified as WUI "archetypes"). That is, we found significant differences in

541 theratings of social context factors across communities at risk from wildfire across nine states. Those

542 findingsalso provide preliminary evidence that large-sample, rapid rural assessments could eventually be

543 useful in existing wildfire planning or strategy at state or federal levels as a means to develop better

544 social data regarding diverse influences on wildfire risk and adaptation (Ojerio et al. 2011; Adger et al.

545 2015; Nielsen Pincus et al. 2015).As we stated above, our use of factor and cluster analyses were chosen

546 to explore a more manageable set ofvariables and to explore existing conceptual ideas (Paveglio et al.

$5472012 ; 2015)$. Comprehensive assessments should test the full range of characteristics used here and

548 consider their impact on a range of adaptive actions in order for more accurate comparisons to existing

549 archetypes.Any data collection surrounding those efforts should recognize how the combination of

550 social context in any given area might differentially influence a variety of actions being promoted to

551 advance FAC, and their variable utility in each of those contexts. Another avenue is the self-assessment

552 tool currently being piloted by the Fire Adapted Communities Learning Network (Fire Adapted 
553 Communities Learning Network 2015). A critical consideration will be the scale at which to consider

554 populations as functional units that will influence one another in the collective management of

555 resources that influence wildfire risk and exposure (Jakes et al. 2011; Williams et al. 2012).Another

556 avenue for future work concerns exploring the influence that extra-local (e.g. county, state or federal)

557 policies, incentives or relationships have on local expression of characteristics. That is, there is a need to 558 explore the extent to which the multi-level structure of wildfire or emergency management enables or 559 constrains local adaptation.

$$
\text { We found significant differences in social context indices across respondent groupings }
$$

createdthrough cluster analysis.Further, analysis of key informant agreement with the effectiveness of

562 community organizing to address wildfire risk shows that the diverse community profiles correspond

563 with key informant agreement about effective community organizing to address wildfire risk. These

564 results give credence to a growing number of scholars and policymakers who describe the WUI as a

565 socially diverse spatial designation rather than a homogeneous collection of people (Brenkert-Smith

566 2011; Collins 2012; Nielsen-Pincus et al. 2015).It corroborates previous assertions that we need to think

567 about at-risk populations as unique cultures that emerge from the intersection of their experiences, 568 social makeup, preferences, and perspectives (Flint and Luloff 2005; Petty et al. 2015; Paveglio et al. 569 2015b).

There remains a significant need to better understand how the characterization of social context

571 will actually influence adaptationto wildfire, which we define as the differential development or

572 adoption of programs, policies and actions aimed at improving local peoples' ability to live with fire

573 given their unique local circumstances. This includes the relative weighting of social context

574 characteristics as more or less important to collective action in the face of wildfire risk, and the

575 development of tailored programs that are most likely to be adopted by unique populations. 
Although our research has advanced understanding of community adaptation to wildfire risks,

577 we recognize a number of additional limitations. For one, our use of key informant assessments in each

578 community could lead to a number of biases. For example, we may have failed to include participants

579 with the knowledge to answer the range of social context questions asked. For these reasons, future

580 research may attempt to recruit a broader range and larger numbers of potential respondents for each

581 community from which rigorous testsof internal consistency and more detailed descriptions of social

582 context characteristics could be applied (Krannich and Humphrey 1986). Lessons from those expanded

583 samples could then be used to develop hierarchical models that account for both informant-level and

584 community-level variation. Reliability tests across respondents and clearer (or more complex)

585 parameters for measurement of possible adaptive actionswould both help ensure the reliability of

586 comparison across cases.

587 Defining community has always been both a challenge and a critically important facet of

588 understanding collective action (Flint et al. 2010; Paveglio et al. 2016). We chose to perform factor and

589 cluster analyses using respondents as the unit of analysis. This was done because it is often difficult to

590 pre-distinguish unique and uniform populations with professionals during the course of a structured

591 interview. Future work should attempt to pre-define with local stakeholders the area populations that

592 constitute unique communities and which would need to be evaluated as different units using our social

593 context measures.

594

595

596 5. Conclusions

The results of our analysisprovide a step forward in the conceptual and methodological

exploration for how diverse elements of social context contribute to wildfire adaptation. Indicator 
context across large samples of human populations, and allow one means for understanding how

601 different combinations of local context might influence support for or enactment of different wildfire

602 management strategies. Promotion of self-assessment approaches such as the one outlined here can

603 help provide indicators across large samples given that in-depth data collection across large numbers of

604 communities on a relatively narrow topic like wildfire adaptation is difficult and time consuming.

605 Despite promoting indicator approaches, we also feel it is important to stress that the goal of

606 understanding is complementary, yet distinct from that ofdocumentation or prediction. Characterizing

607 social context via replicable indicators is only a small initial step in the larger problem-solving process.

608 The combination of local factors that give rise to local culture, and the ways those factors differentially

609 combine to produce adaptive actions, also must be continually informedby select in-depth case studies

610 and collaborative research with members of diverse WUI communities. WUI communities continue to

611 change rapidly, and policymakers must understand their changing (or stable) local cultures if they hope

612 to aid them in becoming "fireadapted." Understanding how local culture and wildfire risk co-evolve

613 comes from systematically exploring how people became who they are, how wildfire intersects with the

614 places they care about, and how different communities are responding to emerging risks. 


\section{References}

Ager, A.A., Kline, J.D., Fischer, A.P. 2015. Coupling the biophysical and social dimensions of wildfire risk to improve wildfire mitigation planning. Risk analysis 35(8): 1393-1406.

Abrams, J.M., M. Knapp, T. Paveglio, A. Ellison, C. Moseley, M. Nielsen-Pincus, M. Carroll. 2015. Re-envisioning community-wildfire relations in the U.S. West as adaptive governance. Ecology \& Society, 20(3): 34.

Absher, J.D., J.J. Vaske. 2011. The role of residents' fire wise actions. International Journal of Wildland Fire, 20: 318-325.

Brenkert-Smith, H. 2010. Building bridges to fight fire: the role of informal social interactions in six Colorado wildland-urban interface communities. International Journal of Wildland Fire 19(6): 689-697.

Brenkert-Smith H. 2011. Homeowners' perspectives on the parcel approach to wildland fire mitigation: the role of community context in two Colorado communities. Journal of Forestry 109(4): 193200

Bryman, A. 2012.Social Research Methods: $4^{\text {th }}$ Edition. New York: Oxford University Press.

Busby, G., Amacher G.S., Haight, R.G. 2013. The social costs of homeowner decision in fireprone communities: Information, insurance, and amenities. Ecological Economics, 92: 104-113.

Johnson Gaither, C., Goodrick, S., Murphy, B.E., Poudyal, N. 2015. An exploratory spatial analysis of social vulnerability and smoke plume dispersion in the U.S. South. Forests (6): 13971421.

Champ, P.A., Brenkert-Smith, H. 2015. Is seeing believing? Perceptions of wildfire risk over time. Risk Analysis. Available at: http://onlinelibrary.wiley.com/doi/10.1111/risa.12465/abstract

Charmaz, K. 2000. Grounded theory: objectivist and constructivist methods. In: Denzin NK, Lincoln YS (eds.) Handbook of QualitativeResearch, 2nd ed. Sage, Thousand Oaks, California, pp 509-535.

Cohn, P.J., Williams, D.R., Carroll, M.S. 2008. Wildland-urban interface residents' views on risk and attribution. P 23-43 in Wildfire risk: human perceptions and management implications, W.E. Martin, C. Raish, B. Kent (eds). Washington, DC: Resources for the Future.

Collins, T.2012. A landscape typology of residential wildfire risk.Pp. 33-65 in D. Paton and F. Tedim (eds.), Wildfire and Community: Facilitating Preparedness and Resilience. Springfield, Il: Charles C. Thomas Publisher.

Cronbach L. 1951. Coefficient alpha and the internal structure of tests. Psychometrika 16.

Diaz, J., Steelman, T., Nowell, B. 2016. Local Ecologi al Knowledge and Fire Management: What does the public understand? Journal of Forestry 114(1): 58-65. 
Dickinson, K., Brenkert-Smith, H., Champ, P., Flores, N. 2015. Catching Fire? Social Interactions, beliefs, and wildfire risk mitigation behaviors. Society \& Natural Resources 28: 807-824.

Dunlop, P.D., McNeill, I.M., Boylan, J.L., Morrison, D.L., Skinner, T.C. 2014. Preparing ... for what? Developing multi-dimensional measures of community wildfire preparedness for researchers, practitioners and households. International Journal of Wildland Fire, 23(6): 887-896.

Everitt BS. 1980. Cluster Analysis, 2nd Edition. London: Heineman Educational Books.

Eyler, A.A., Mayer, J., Rafii, R., Housemann, R., Brownson, R.C., King, A. 1999. Key informant surveys as a tool to implement and evaluate physical activity interventions in the community. Health Education Research 14(2): 289-298.

Fire Adapted Communities Coalition (FACC). 2015. Fire adapted communities. http://www.fireadapted.org

Fischer, A.P., Kline, J.D., Charnley, S., Olsen, C. 2012. Identifying policy target groups with qualitative and quantitative methods: The case of wildfire risk on nonindustrial private forest lands. Forest Policy and Economics 25: 62-71.

Flint, C.G., Luloff, A.E. 2005. Natural resource-based communities, risk, and disaster: An Intersection of Theories. Society and Natural Resources 18(5): 399-412.

Flint, C.G., Luloff A.E., Theodori G. 2010. Extending the concept of community interaction to explore regional community fields.Journal of Rural Social Science 25(1): 1-13.

Ford, J.D., Pearce, T., Duerden, F., FurgalC., Smit, B. 2010. Climate change policy responses for Canada's Inuit population: The importance of and opportunities for adaptation. Global EnvironmentalChange20: 177-191.

Gordon, J.S., Luloff A., Stedman R.C. 2012. A multisite qualitative comparison on community wildfire risk perceptions. Journal of Forestry 110(2): 74-78.

Homburg, C., Klarmann, M., Reimann, M., Schilke, O. 2012. What drives key informant accuracy? Journal of Marketing Research 49: 594-608.

International Association of Wildland Fire Chiefs (IAFC). 2015. Ready, Set, Go! Available at: http://www.wildlandfirersg.org/

Jakes, P.J., V. Sturtevant. 2013. Trial by fire: Community Wildfire Protection Plans put to the test. International Journal of Wildland Fire228(8): 1134-1143.

Jakes, P.J., E.R. Langer. 2012. The adaptive capacity of New Zealand communities to wildfire. International Journal of Wildland Fire 21(6), 764-772.

Jakes P.J., Nelson K.C., Enzler S.A., Burns S., Cheng A.S., Sturtevant V., Williams D.R., Bujak A., 
Brummel R.F., Grayzeck-Souter S. 2011. Community wildfire protection planning: is the Healthy Forests Restoration Act's vagueness genius? International Journal of Wildland Fire 20: 350-363.

Jakes, P.J., L. Kruger, M. Monroe, K. Nelson, and V. Strurtevant. 2007. Improving wildfire preparedness: lessons from communities across the U.S. Human Ecology Review 14(2): 188-197.

Kulig, J.C., D.S. Edge, I. Townshend, N. Lightfoot, and W. Reimer. 2013. Community resiliency: Emerging theoretical insights. Journal of Community Psychology. 41(6): 758-775.

Krannich R.S., Humphrey C.R. 1986. Using Key Informant Data in Comparative Community Research An Empirical Assessment. Sociological methods \& research 14: 473-493.

Kyle, G.T., Theodori, G.L., Absher, D., Jun. J. 2010. The influence of home and community attachment on firewise behavior. Society and Natural Resources 23(11): 1075-1092.

Lee, R.G. 1991. Four myths of interface communities: rural localities do not epitomize idealized conceptions. Journal of Forestry 89(6): 35-38.

Lindlof, T.R., and B.C. Taylor. 2010. Qualitative communication research methods: Third edition. Thousand Oaks, CA, Sage.

Martin, W.E., Martin, I.M., Kent, B. 2009. The role of risk perceptions in the risk mitigation process: The case of wildfire in high risk communities. Journal of Environmental Management: 91(2): 489-498.

McCaffrey, S. 2015. Community wildfire preparedness: a global state-of-the-knowledge summary of social science research. Current Forestry Reports 1: 81-90.

McCaffrey, S. and C.C. Olsen. 2012. Research perspectives on the public and fire management: a synthesis of current social science on eight essential questions. USDA For. Serv. Gen. Tech. Rep. NRS-GTR-104.40 p.

McCaffrey, S.M., M. Stidham, E. Toman, B. Shindler. 2011. Outreach programs, peer pressure, and common sense: What motivates homeowners to mitigation wildfire risk? Environmental Management 48(3): 475-488.

Meldrum, J.R., P.A. Champ, T. Warziniack, H. Brenkert-Smith, C.M. Barth and L. C. Falk. 2014. Cost shared wildfire risk mitigation in Log Hill Mesa, Colorado: survey evidence on participation and willingness to pay. International Journal of Wildland Fire 23: 567-576.

Monroe, M.C., Ballard, H.L., Oxarart, A., Sturtevant, V.E., Jakes, P.J., Evans, E.R. 2015. Agencies, educators, communities and wildfire: partnerships to enhance environmental education for youth. Environmental Education Research. Available online at: http://www.tandfonline.com/doi/abs/10.1080/13504622.2015.1057555?journalCode=ceer20

National Fire Protection Association. 2015. Firewise Communities USA/Recognition Program. Available at: http://www.firewise.org/ 
Nielsen-Pincus M., Ribe R.G., Johnson B.R. 2015. Spatially and socially segmenting private landowner motivations, properties, and management: A typology for the wildland urban interface. Landscape and Urban Planning 137: 1-12.

Norris, F.H., Stevens, S.P., Pfefferbaum, B., Wyche, K.F., Pffefferbaum, R.L. 2008. Community resilience as a metaphor, theory, set of capacities, and strategy for disaster readiness. American Journal of Community Psychology 41: 127-150.

Ojerio R., Moseley C., Lynn K., Bania N. 2011. Limited involvement of socially vulnerable populations in federal programs to mitigate wildfire risk in Arizona. Natural Hazards Review 12(1): 28-36.

Paton, D., Buergelt, P.T., Campbell, A. 2015. Learning to co-exist with Environmental Hazards: Community and Societal Perspectives and Strategies. In Daniels, J.A. (ed.) Advances in Environmental Research, Vol. 43. Hauppauge, NY. Nova Science Publishers.

Paveglio, T.B.,J. Abrams, and A. Ellison.2016. Developing fire adapted communities: The importance of interactions among elements of local context. For publication in Society \& Natural Resources.

Paveglio, T.B.,H.Brenkert-Smith, T. Hall, A.S. Smith. 2015a. Understanding social impact from wildfires: advancing means for assessment. The International Journal of Wildland Fire 24(2):212-224.

Paveglio,T.B., C. Moseley, M.S. Carroll, D.R. Williams, A.P. Fischer, E.J. Davis. 2015b. Categorizing the social context of the Wildland Urban Interface: Adaptive capacity for wildfire and community "archetypes."Forest Science, 61(2): 298-310.

Paveglio, T.B., M.S. Carroll, P.J Jakes and T. Prato. 2012. Exploring the social characteristics of adaptive capacity to wildfire: Insights from Flathead County, Montana. Human Ecology Review, 19(2), 110-124.

Paveglio, T.B., P.J. Jakes, M.S. Carroll and D.R. Williams. 2009. Understanding social complexity within the wildland urban interface: A new species of human habitation? Environmental Management, 43, 1085-1095.

Petty, A.M., Isendahl, C., Brenkert-Smith, H., Goldstein, D.J., Rhemtulla, J.M., Rahman, S.A., Kumasi, T.C. 2015. Applying historical ecology to natural resource management institutions:Lessons from two case studies of landscape fire management. Global Environmental Change 31: 1-10.

Poudyal N.C., Johnson-Gaither C., Goodrick S., Bowker J.M., Gan J. 2012. Locating spatial variation in the association between wildland fire risk and social vulnerability across six southern states. Environmental Management 49, 623-635.

Pyne, S. 2015. Between Two Fires: A Fire History of Contemporary America. University of Arizona press.522 p. 
Sharp E.A., Thwaites R., Curtis A., Millar J. 2013. Factors affecting community-agency trust before, during and after a wildfire: An Australian case study. Journal of Environmental Management 130: 10-19.

Sherrieb, K., Louis, C.A., Pfefferbaum, R.L., J.D. Pfefferbaum B., Diab, E., Norris, F.H. 2012. Assessing community resilience on the US coast using school principals as key informants. International Journal of Disaster Risk Reduction 2: 6-15.

Short, J.F. 1984. "The Social Fabric at Risk: Toward the Social Transformation of Risk Analysis."American Sociological Review: 711-725.

Solangaarachichi, D., Griffin, A.L., Doherty, M.D. 2012. Social vulnerability in the context of bushfire risk at the urban-bush interface in Sydney: a case study of the Blue Mountains and Kuring-gai local council areas. Natural Hazards 64: 1873-1898.

Spies, T.A., E. M. White, J. D. Kline, A.P. Fischer, A. Ager, J. Bailey, J. Bolte, J. Koch, E. Platt, C.S. Olsen, D. Jacobs, B. Shindler, M.M. Steen-Adams, and R. Hammer. 2014. Examining fireprone forest landscapes as coupled human and natural systems. Ecology\& Society.19(3): 9.

Steelman T.A., McCaffrey S.2013. Best practices in risk and crisis communication: Implications for natural hazards management. Natural Hazards 65: 683-705.

Stevens J.P. 2009. Applied Multivariate Statistics for the Social Sciences, 5th ed. Routledge, Taylor \& Francis Group, New York.

Stidham, M., S. McCaffrey, E. Toman, and B. Shindler. 2014. Policy tools to encouragecommunity level defensible space in the United States: A tale of six communities. Journal of Rural Studies 35: 59-69.

Sword-Daniels, V., Eriksen, C., Hudson-Doyle, E.E., Alaniz R., Adler, C., Schenk, T., Vallance, S. 2016. Embodied uncertainty: living with complexity and natural hazards. Journal of Risk Research. Available at: http://www.tandfonline.com/doi/full/10.1080/13669877.2016.1200659

Toman, E., S. Melanie, S. McCaffrey, and B. Shindler. 2013. Social science at the wildlandurban interface: a compendium of research results to create Fire-Adapted Communities. USDA For. Serv. Gen. Tech. Rep. NRS-GTR-111.75 p.

USDA Forest Service, National Fire and Aviation Management: Web applications. Available at: https://fam.nwcg.gov/fam-web/

Wagner, S.M., Rau, C., Lindemann, E. 2010. Multiple informant methodology: A critical review and recommendations. Sociological Methods \& Research 38(4): 582-618.

Ward, J.H. 1963. Hierarchical grouping to optimize an objective function.Journal of the American Statistical Association 58: 236-244.

Wildland Fire Executive Council (WFEC).2014 . The national strategy: The final phase in the 
development of the National Cohesive Wildland Fire Management Strategy.

http://www.forestsandrangelands.gov/leadership/WFEC/index.shtml

Williams, D.R., P.J. Jakes, S. Burns, A.S. Cheng, K.C. Nelson, V. Sturtevant, R.F. Brummel, E. Staychock, and S.G. Souter. 2012. Community Wildfire Protection Planning: The importance of framing, scale and building sustainable capacity. Journal of Forestry 110(8): 415-420.

\section{List of Tables}

Table 1.Key informantsurvey responses by state

Table 2.Factor analysis solution based onkey informant structured interview responses about social context in wildland urban interface communities

Table 3.Results of multivariate regression for influence of social context indices on key informant rating of community adaptive actions.

Table 4.Key informant structured interview clusters (panel A), and profiles for each cluster; cluster mean values computed from least squares one-way ANOVA results indicate significant differences among cluster means(panel B).

Appendix 1. Responses to key informant structured interview statements about social context in wildland urban interface communities. 
Table 1.Key informant survey responses by state

\begin{tabular}{lc}
\hline State & $\begin{array}{c}\text { Key informant } \\
\text { responses }\end{array}$ \\
\hline AZ & 14 \\
CA & 15 \\
CO & 4 \\
ID & 18 \\
MT & 10 \\
NM & 10 \\
OR & 34 \\
UT & 5 \\
WA & 5 \\
\hline Total & 115
\end{tabular}


Table 2 -Factor analysis solution based onkey informant structured interview statements about social context in wildland urban interface communities (factor loadings greater than 0.40 bolded for emphasis).

\begin{tabular}{|c|c|c|c|c|c|}
\hline \multirow[b]{2}{*}{ Key Informant Interview Statements } & \multicolumn{4}{|c|}{ Varimax Rotated Factor Loadings } & \multirow{2}{*}{$\begin{array}{l}\text { Chronbach's } \\
\text { Alpha }\end{array}$} \\
\hline & Factor 1 & Factor 2 & Factor 3 & Factor 4 & \\
\hline Factor 1-Social Cohesion & & & & & 0.89 \\
\hline Residents share a sense of values and culture & 0.89 & 0.11 & -0.11 & 0.06 & \\
\hline Residents have a strong sense of community & 0.82 & 0.09 & -0.02 & 0.11 & \\
\hline Residents are vested in the future and well-being of each other & 0.80 & 0.18 & -0.01 & 0.03 & \\
\hline Factor 2 - Place-Based Knowledge & & & & & 0.76 \\
\hline $\begin{array}{l}\text { People in these at-risk areas tend to consider wildfire risk reduction to be } \\
\text { someone else's problem* }\end{array}$ & 0.08 & 0.71 & 0.00 & 0.21 & \\
\hline People in these at-risk areas understand wildfire risk in the area & 0.19 & 0.68 & -0.04 & 0.18 & \\
\hline $\begin{array}{l}\text { People in these at-risk areas understand the steps they can take to reduce } \\
\text { wildfire risk }\end{array}$ & 0.09 & 0.67 & -0.03 & 0.20 & \\
\hline People in these at-risk areas have detailed understanding of area fire regimes & 0.04 & 0.51 & -0.02 & 0.07 & \\
\hline Factor 3 - Access to Scientific and Technical Knowledge & & & & & 0.77 \\
\hline $\begin{array}{l}\text { There are individuals in the community with emergency management or } \\
\text { wildland firefighting experience }\end{array}$ & -0.11 & 0.01 & 0.80 & -0.15 & \\
\hline There are residents in the community with grant writing experience & 0.10 & -0.04 & 0.66 & -0.04 & \\
\hline There are individuals in the community with knowledge about the local ecology & -0.22 & -0.04 & 0.65 & -0.10 & \\
\hline $\begin{array}{l}\text { Local leaders spearhead efforts to plan for and implement projects to reduce } \\
\text { impacts from wildfires }\end{array}$ & 0.04 & -0.04 & 0.60 & 0.01 & \\
\hline Factor 4 - Shared Responsibility and Understanding & & & & & 0.70 \\
\hline $\begin{array}{l}\text { New residents in these at-risk areas are equally as interested in mitigating } \\
\text { wildfire risk as long-time residents }\end{array}$ & 0.10 & 0.19 & -0.08 & 0.82 & \\
\hline $\begin{array}{l}\text { Second homeowners and seasonal residents in these at-risk areas are equally as } \\
\text { interested in mitigating wildfire risk as other residents }\end{array}$ & 0.01 & 0.24 & -0.07 & 0.62 & \\
\hline $\begin{array}{l}\text { People in these at-risk areas understand that there may not be enough } \\
\text { firefighting resources to save every home at risk from wildfire }\end{array}$ & 0.10 & 0.31 & -0.11 & 0.41 & \\
\hline
\end{tabular}




\section{Factor Solution}

Initial eigenvalues of correlation matrix

$\begin{array}{llll}3.77 & 2.25 & 2.01 & 1.16\end{array}$

Proportion of total variance explained by retained factors

$27 \% \quad 16 \% \quad 14 \% \quad 8 \%$

Eigenvalues of the reduced correlation matrix

$\begin{array}{llll}3.34 & 1.76 & 1.65 & 0.68\end{array}$

Proportion of common variance explained by retained factors

$45 \% \quad 24 \% \quad 22 \% \quad 9 \%$

* Items were reverse coded for consistency in factor analysis. 
Table 3.Results of multivariate regression for influence of social context indices on key informant rating of community adaptive actions.

\begin{tabular}{|c|c|c|c|c|c|c|c|c|}
\hline \multirow[b]{3}{*}{ Independent variables } & \multirow{2}{*}{\multicolumn{2}{|c|}{$\begin{array}{c}\text { The community has } \\
\text { organized effectively } \\
\text { to address wildfire } \\
\text { risk }\end{array}$}} & \multicolumn{2}{|c|}{$\begin{array}{c}\text { The community has } \\
\text { organized effectively } \\
\text { to address wildfire } \\
\text { risk }\end{array}$} & \multirow{2}{*}{\multicolumn{2}{|c|}{$\begin{array}{l}\text { The community has } \\
\text { implemented a } \\
\text { publicity campaign } \\
\text { about reducing } \\
\text { wildfire risk on private } \\
\text { property } \\
\text { Main Effects Model }\end{array}$}} & \multicolumn{2}{|c|}{$\begin{array}{l}\text { The community has } \\
\text { implemented a } \\
\text { publicity campaign } \\
\text { about reducing } \\
\text { wildfire risk on private } \\
\text { property }\end{array}$} \\
\hline & & & Interact & n Model & & & Interactio & Model \\
\hline & $\begin{array}{c}b^{a} \\
(S E)\end{array}$ & $\beta^{b}$ & $\begin{array}{l}b^{a} \\
(S E)\end{array}$ & $\beta^{b}$ & $\begin{array}{c}b^{a} \\
(S E)\end{array}$ & $\beta^{b}$ & $\begin{array}{l}b^{a} \\
(S E)\end{array}$ & $\beta^{b}$ \\
\hline \multirow[t]{2}{*}{ Shared responsibility and understanding } & -0.12 & -0.09 & -0.47 & -0.38 & 0.05 & 0.04 & $0.73 *$ & 0.60 \\
\hline & $(0.10)$ & & $(0.32)$ & & $(0.11)$ & & $(0.35)$ & \\
\hline \multirow[t]{2}{*}{ Social cohesion } & $0.32 * *$ & 0.22 & -0.22 & -0.15 & -0.07 & -0.05 & -0.01 & -0.01 \\
\hline & $(0.11)$ & & $(0.26)$ & & $(0.12)$ & & $(0.27)$ & \\
\hline \multirow[t]{2}{*}{ Place-based knowledge } & $0.33 * *$ & 0.23 & $1.14^{* *}$ & 0.80 & 0.20 & 0.15 & 0.56 & 0.40 \\
\hline & $(0.12)$ & & $(0.35)$ & & $(0.13)$ & & $(0.38)$ & \\
\hline \multirow[t]{2}{*}{ Access to scientific and technical knowledge } & $0.96 * * *$ & 0.60 & $0.54^{*}$ & 0.34 & $0.80 * * *$ & 0.52 & $0.99 * * *$ & 0.64 \\
\hline & $(0.12)$ & & $(0.26)$ & & $(0.13)$ & & $(0.29)$ & \\
\hline \multirow[t]{2}{*}{ Place-based knowledge X Social cohesion } & & & -0.31 & 0.04 & & & -0.24 & -0.27 \\
\hline & & & $(0.18)$ & & & & $(0.20)$ & \\
\hline Place-based knowledge $X$ Access to & & & $-0.34^{*}$ & -0.31 & & & -0.00 & -0.00 \\
\hline scientific and technical knowledge & & & $(0.15)$ & & & & $(0.17)$ & \\
\hline Place-based knowledge X Shared & & & -0.02 & -0.02 & & & -0.02 & -0.02 \\
\hline responsibility and understanding & & & $(0.12)$ & & & & $(0.13)$ & \\
\hline \multirow[t]{2}{*}{ Social cohesion X Access to knowledge } & & & $0.41 *$ & 0.50 & & & -0.01 & -0.01 \\
\hline & & & $(0.19)$ & & & & $(0.21)$ & \\
\hline Social cohesion X Shared responsibility and & & & 0.31 & 0.36 & & & -0.21 & -0.25 \\
\hline understanding & & & $(0.17)$ & & & & $(0.18)$ & \\
\hline Access to scientific and technical knowledge & & & 0.31 & -0.02 & & & $-0.38^{*}$ & -0.42 \\
\hline X Shared responsibility and understanding & & & $(0.17)$ & & & & $(0.17)$ & \\
\hline
\end{tabular}


Dependent Variable Mean

$\mathrm{n}$

Model F-value

$\mathrm{R}^{2}$

Adjusted $\mathrm{R}^{2}$

${ }^{\mathrm{a} U n s t a n d a r d i z e d}$ regression coefficient

${ }^{b}$ Standardized regression coefficient

${ }^{*} p<0.05 ;{ }^{* *} p<0.01 ;{ }^{* * *} p<0.001$

\begin{tabular}{cccc}
0.56 & 0.56 & 0.72 & 0.72 \\
114 & 114 & 114 & 114 \\
$21.85^{* * *}$ & $10.30^{* * *}$ & $10.80^{* * *}$ & $5.74^{* * *}$ \\
0.45 & 0.50 & 0.28 & 0.36 \\
0.42 & 0.45 & 0.26 & 0.30 \\
\hline
\end{tabular}


Table 4. Key informant structured interview clusters (panel A), and profiles for each cluster; cluster mean values computed from least squares one-way ANOVA results indicate significant differences among cluster means (panel B).

\begin{tabular}{|c|c|c|c|c|}
\hline $\begin{array}{l}\text { Panel A. } \\
\text { Cluster } \\
\text { Number }\end{array}$ & \multicolumn{2}{|c|}{$\begin{array}{l}\text { Number of } \\
\text { Respondents per Cluster }\end{array}$} & \multicolumn{2}{|c|}{$\begin{array}{l}\text { Percent of } \\
\text { Respondents per cluster }\end{array}$} \\
\hline 1 & 48 & & 42 & \\
\hline 2 & 26 & & 23 & \\
\hline 3 & 20 & & 17 & \\
\hline \multirow[t]{2}{*}{4} & 21 & & 18 & \\
\hline & 115 & & 100 & \\
\hline \multicolumn{5}{|l|}{ Panel B. } \\
\hline & $\begin{array}{l}\text { Place-based } \\
\text { knowledge }\end{array}$ & $\begin{array}{l}\text { Access to } \\
\text { Scientific and } \\
\text { Technical } \\
\text { Knowledge }\end{array}$ & $\begin{array}{l}\text { Social } \\
\text { Cohesion }\end{array}$ & $\begin{array}{l}\text { Shared } \\
\text { Responsibility } \\
\text { and } \\
\text { Understanding }\end{array}$ \\
\hline \multicolumn{5}{|c|}{ Mean values for each cluster } \\
\hline Cluster 1 & $0.14^{\mathrm{A}}$ & $1.56^{\mathrm{A}}$ & $1.28^{\mathrm{A}}$ & $-0.02^{A}$ \\
\hline Cluster 2 & $0.93^{B}$ & $1.33^{\mathrm{B}}$ & $1.46^{\mathrm{A}}$ & $1.35^{\mathrm{B}}$ \\
\hline Cluster 3 & $-0.34^{C}$ & $1.13^{\mathrm{B}}$ & $0.83^{\mathrm{B}}$ & $-0.98^{C}$ \\
\hline Cluster 4 & $-0.38^{A}$ & $0.35^{c}$ & $0.87^{\mathrm{B}}$ & $0.62^{\mathrm{D}}$ \\
\hline \multicolumn{5}{|c|}{ ANOVA Statistics } \\
\hline $\mathbf{N}$ & 115 & 115 & 115 & 115 \\
\hline F-statistic & $18.06 * * *$ & $32.23 * * *$ & $5.06 * *$ & $103.04 * * *$ \\
\hline R-square & 0.33 & 0.47 & 0.12 & 0.74 \\
\hline
\end{tabular}


Appendix 1. Responses to key informant structured interview statements about social context in wildland urban interface communities (number of responses in parenthesis. Item responses ranged from strongly agree (2) to strongly disagree (-2), with a midpoint labeled neither agree nor disagree (0).

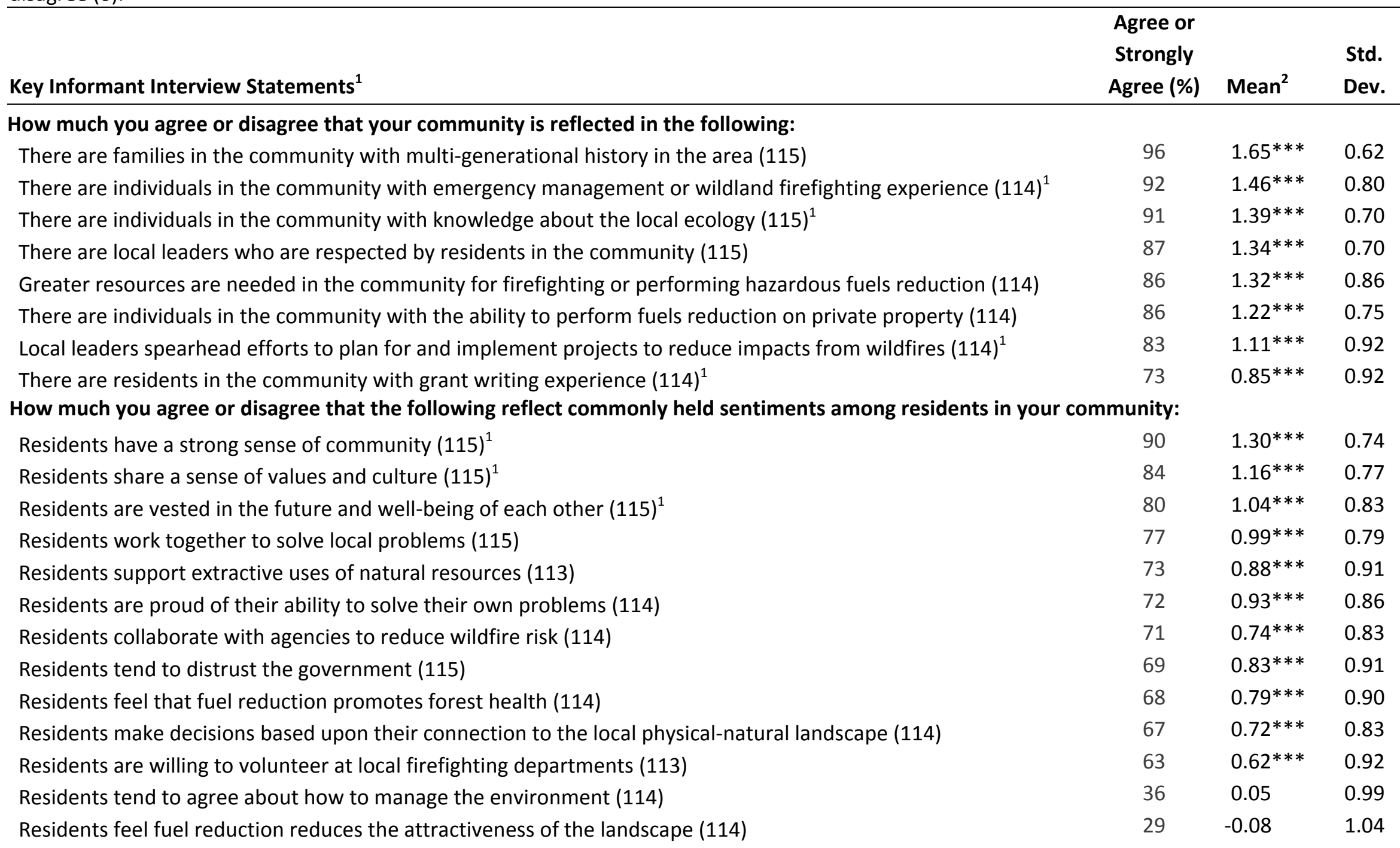

How much you agree or disagree that the following reflect resident preparation for wildfire in high risk areas of your community: 
People in at-risk areas understand wildfire risk in the area $(115)^{1}$

People in at-risk areas receive and share information about wildfire through agency communication or specialized wildfire programs like FireWise (112)

People in at-risk areas understand the steps they can take to reduce wildfire risk (115) ${ }^{1}$

People in at-risk areas have the ability to mitigate wildfire risk themselves (114)

People in at-risk areas tend to consider wildfire risk reduction to be someone else's problem (114) ${ }^{1}$

People in at-risk areas receive and share information about wildfire through family and friends (115)

Of those with financial means, people living in at-risk areas are willing to spend money to mitigate wildfire risk (113)

People in at-risk areas tend to consider wildfire risk reduction to be their own problem (114)

People in at-risk areas have the financial means to mitigate wildfire risk (114)

People in at-risk areas have detailed understanding of area fire regimes (114) ${ }^{1}$

How much you agree or disagree with the following regarding firefighting resources inyour community:

Your community has local firefighters that are well-trained for wildland fire (114)

Your community has local firefighters that are well-trained for structural fire (113)

Your community has sufficient resources to manage wildfire risk in the area (115)

How much you agree or disagree with the following in high risk areas of your community:

People in at-risk areas understand that there may not be enough firefighting resources to save every home at risk from wildfire (114) $)^{1}$

New residents in at-risk areas are equally as interested in mitigating wildfire risk as long-time residents (114) ${ }^{1}$

People in at-risk areas do not feel that wildfire will impact them (114)

Second homeowners and seasonal residents in at-risk areas are equally as interested in mitigating wildfire risk as other residents $(115)^{1}$

How much you agree or disagree with the following about business and civic climate in your community:

Your community has an active set of local civic organizations (113)

Your community has an active community wildfire organization (113)

Your community has an active local biomass or wood products industry (114)

\begin{tabular}{lll}
78 & $0.81^{* * *}$ & 0.87 \\
74 & $0.79^{* * *}$ & 0.72 \\
72 & $0.76^{* * *}$ & 0.79 \\
62 & $0.59^{* * *}$ & 0.93 \\
53 & $0.35^{* * *}$ & 1.08 \\
51 & $0.42^{* * *}$ & 0.75 \\
41 & $0.18^{*}$ & 0.88 \\
32 & -0.05 & 0.87 \\
30 & -0.15 & 0.95 \\
28 & -0.13 & 1.02 \\
& & \\
85 & $1.11^{* * *}$ & 0.88 \\
82 & $1.10^{* * *}$ & 0.91 \\
40 & $-0.24^{*}$ & 1.21 \\
& & \\
54 & $0.32^{* *}$ & 1.06 \\
& & \\
41 & $0.25^{* *}$ & 0.98 \\
35 & -0.08 & 1.11 \\
32 & -0.01 & 1.07 \\
& & \\
72 & $0.67 * * *$ & 1.20 \\
57 & $0.46^{* * *}$ & 1.20 \\
26 & $-0.48^{* * *}$ & 1.21 \\
\hline
\end{tabular}

1. Superscript " 1 " indicates statements that were retained in the factor analysis

2. Asterisks indicate probability level that mean values are different than zero (neither agree nor disagree); ${ }^{*} p<0.10 ;{ }^{* *} p<0.01 ;{ }^{* * *} p<0.001$ 